\title{
Correction to: 3D-Printed Poly(E-aprolactone)/Graphene Scaffolds Activated with P1-Latex Protein for Bone Regeneration, by Caetano GF, Wang W, Chiang W-H, Cooper G, Diver C, Blaker JJ, Frade MA, and Bártolo P. 3D Print Addit Manuf 2018;5(2):127-137. DOI: 10.1089/3dp.2018.0012
}

In the June 2018 IsSUe of $3 D$ Printing and Additive Manufacturing (vol. 5, no. 2, pp. 127-137), in the article entitled "3DPrinted Poly( $\varepsilon$-aprolactone)/Graphene Scaffolds Activated with P1-Latex Protein for Bone Regeneration," by Caetano GF et al., the word "trabecular" was used in three separate locations where "cortical" should have been used.

The first was on page 128, in Figure 1e.

Figure 1e originally appeared as below on the left. The corrected Figure 1e appears on the right.

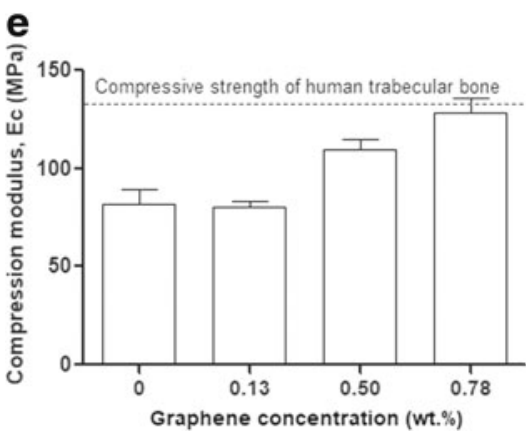

FIG. 1e. Original.

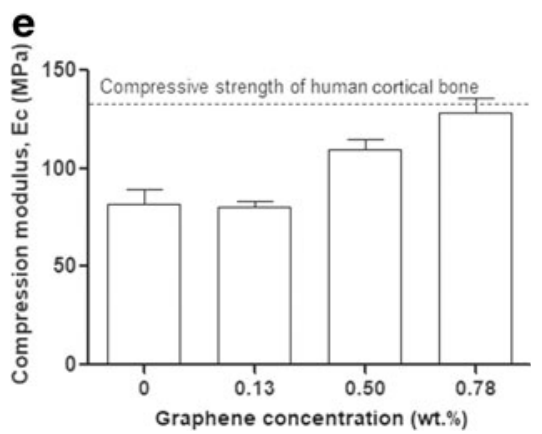

FIG. 1e. Corrected.

The second case was originally written as: "Moreover, maximum values of compressive modulus observed for scaffolds containing higher concentrations of graphene are in the midrange of properties reported for human trabecular bone. ${ }^{31,}$

The correct sentence reads: "Moreover, maximum values of compressive modulus observed for scaffolds containing higher concentrations of graphene are in the midrange of properties reported for human cortical bone. ${ }^{31,}$

The third occurrence was written as: "As a strong material, the addition of even small graphene levels to PCL scaffolds improved mechanical properties, with scaffolds containing $0.78 \mathrm{wt}$ \% presenting a compressive modulus close to the trabecular bone."

The corrected sentence now reads: "As a strong material, the addition of even small graphene levels to PCL scaffolds improved mechanical properties, with scaffolds containing $0.78 \mathrm{wt} . \%$ presenting a compressive modulus close to the cortical bone."

The online version of the article has been corrected to reflect these changes.

The authors wish to apologize for the error. 